\title{
Slip Control of Electric Vehicle Based on Tire-Road Friction Coefficient Estimation
}

\author{
Gaojian Cui, ${ }^{1}$ Jinglei Dou, ${ }^{1}$ Shaosong Li, ${ }^{1}$ Xilu Zhao, ${ }^{2}$ Xiaohui Lu, ${ }^{1}$ and Zhixin Yu \\ ${ }^{1}$ School of Mechatronics Engineering, Changchun University of Technology, Changchun 130012, China \\ ${ }^{2}$ Faculty of Engineering, Saitama Institute of Technology, Fukaya 369-0293, Japan
}

Correspondence should be addressed to Shaosong Li; lishaosong@ccut.edu.cn

Received 24 May 2017; Revised 7 August 2017; Accepted 6 September 2017; Published 7 November 2017

Academic Editor: Filippo Cacace

Copyright (c) 2017 Gaojian Cui et al. This is an open access article distributed under the Creative Commons Attribution License, which permits unrestricted use, distribution, and reproduction in any medium, provided the original work is properly cited.

The real-time change of tire-road friction coefficient is one of the important factors that influence vehicle safety performance. Besides, the vehicle wheels' locking up has become an important issue. In order to solve these problems, this paper comes up with a novel slip control of electric vehicle (EV) based on tire-road friction coefficient estimation. First and foremost, a novel method is proposed to estimate the tire-road friction coefficient, and then the reference slip ratio is determined based on the estimation results. Finally, with the reference slip ratio, a slip control based on model predictive control (MPC) is designed to prevent the vehicle wheels from locking up. In this regard, the proposed controller guarantees the optimal braking torque on each wheel by individually controlling the slip ratio of each tire within the stable zone. Theoretical analyses and simulation show that the proposed controller is effective for better braking performance.

\section{Introduction}

In recent years, as an elegant way to relieve energy consumption as well as emission concerns in the transportation sector, EVs have attracted a large amount of interest [1]. With the motors, respectively, mounted in or nearby each wheels, the motor braking has obvious advantages like fast torque response, higher efficiency, compact structure, and so on.

Wheel slip ratio is an indispensable parameter of the research on vehicle braking. Optimal slip control on braking can reduce braking distance, save braking energy, and enhance the vehicle stability. For these purposes, various methods have been used in slip control, such as sliding mode control [2], robust control [3], and fuzzy control [4]. However, most of these studies are done with the road adhesion coefficient known.

Real-time estimation of adhesion coefficient is an important issue of control strategy's realization and work performance's improvement of vehicle chassis' active safety system. Currently, the estimation methods of road adhesion coefficient mainly include the following: (1) measure those factors which have much influence on adhesion coefficient and forecast value of adhesion coefficient according to experiences, such as detection method of adhesion coefficient based on optical sensor or ultrasonic sensor $[5,6]$; (2) measure the motion response of vehicle body or wheel which is caused by adhesion coefficient; this method can estimate adhesion coefficient according to tire strain, stress, noise, and vehicle longitudinal and lateral dynamics, such as detection method of adhesion coefficient based on curves between adhesion coefficient and slip rate $[7,8]$. The former method's accuracy is high; however it needs additional expensive sensors and depends on previous experience and external environment. The latter method has been widely applied, but its accuracy is low, or performance in real-time is poor.

In addition, slip control has attracted more and more attention up to now. In recent years, kinds of researches and theses show interest in slip control; slip control has been applied in ABS, CSR, and so on and ensured the vehicle stability. A novel control scheme by coordinating the slips of the two wheels on the same side simultaneously was proposed in [9]. Research in [10] proposed a robust adaptive wheel 
slip controller and a wheel torque allocator to distribute the braking effort among the two actuators. An integrated slipbased torque control strategy for regenerative braking and hydraulic antilock braking system was developed according to the logic threshold concept in [11]. Research in [12] put forward a method for the calculation of optimal slip ratio and a novel control strategy with engine controller for traction control system.

However, these studies have limitations in dealing with constrained problem. Nowadays, a control method model predictive control (MPC) has been accepted widely. The main merits of MPC cover the following points: (1) MPC can deal with complex optimization problems as well as handling constraints; (2) it can deal with the multiple control requirements with the weighting matrix being considered and incorporates all control objectives in only one formulation and the control parameters are easy to tune; (3) the timedomain constraints can be taken into account, such as motor maximum limitations and performance metrics [14].

In consideration of these viewpoints, this paper has developed a slip control of EV based on tire-road friction coefficient estimation. This controller based on MPC prevents the vehicle from locking up while braking. When it worked, the maximum tire-road friction coefficient is estimated firstly, and then the reference slip ratio can be addressed. Meanwhile, motor torque output was suppressed by an allowable torque value acquired by friction force observer and reference wheel slip. In addition, due to the fast response of the motor braking system of EV, a better wheel slip control performance can be obtained. Through the simulation case studies and analysis discussions, the effectiveness and adaptability of the proposed control are demonstrated.

The organization of this paper is as follows. In Section 2, the system model of EV is explained briefly, which discusses the vehicle dynamics model while braking and slip ratio model. In Section 3, the proposed tire-road friction coefficient estimation is explained. Section 4 expounds the slip controller based on MPC. In this section, the reference value of MPC is provided based on the maximum tire-road friction coefficient which is calculated from Section 3. In Section 5, simulation results are given to verify the effectiveness of the proposed control system on two road conditions. Finally, the paper ends with conclusions in Section 6.

\section{System Model}

2.1. Vehicle Dynamics Model. Consider a vehicle longitudinal motion when braking operation is performed on a flat road. Ignoring the air resistance and rolling resistance, the single wheel model is shown as in Figure 1.

This model assumes that longitudinal braking conditions and steering effects are neglected, and the dynamic differential equations are described as

$$
\begin{aligned}
& J \dot{\omega}=F_{x} R-T_{b}, \\
& \frac{m}{4} \dot{v}=F_{x},
\end{aligned}
$$

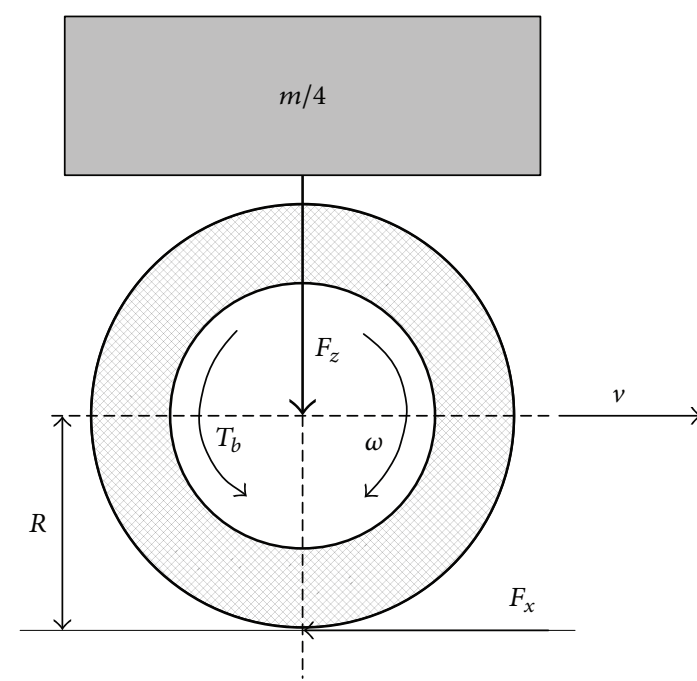

FIGURE 1: Single wheel model of vehicle braking.

$$
\begin{aligned}
& F_{x}=\mu(\lambda) F_{z}, \\
& F_{z}=\frac{m g}{4},
\end{aligned}
$$

where $J$ is the wheel inertia and $\omega$ is the wheel angular speed, $T_{b}$ is the braking torque, $F_{x}$ is the friction force, $R$ is the wheel radius, $m$ is the vehicle mass, and $v$ is the vehicle velocity. In this section, we assume that the driving resistance is considered to be small and ignorable. Moreover, $\mu$ is the tire-road friction coefficient, $\lambda$ is the slip ratio, and $F_{z}$ is the vehicle normal load; in this paper, we assume that the normal load has no big change.

2.2. Longitudinal Slip Ratio Calculation Model. Once braking force applied on the wheel is greater than the friction force that a road surface can supply, the wheel slip will increase to result in wheel lock and cause vehicle instability. The longitudinal slip is obtained by dividing the slip velocity by the velocity of the vehicle

$$
\lambda=\frac{v-\omega R}{\max (v, \varepsilon)}
$$

where $\varepsilon$ is a small constant in order to avoid zero denominator. In this paper, the value of $\varepsilon$ is set as 0.1 .

By taking the differential of (5), the slip dynamics can be expressed as

$$
\dot{\lambda}=\frac{\dot{v}}{v}(1-\lambda)-\frac{\dot{\omega} R}{v} .
$$

\section{Tire-Road Friction Coefficient Estimation}

In this section, an estimation method of tire-road friction coefficient is proposed. The primary task of this method is to make vehicles drive on various road conditions. By designing a novel $\mu$ - $\lambda$ curve based on five accurate road conditions, the peak value point of the curve is confirmed. With this peak value of friction coefficient, the slip control can be continued. 
TABLE 1: Parameters of the tire model for various surfaces confirmed.

\begin{tabular}{lccc}
\hline Surface condition & $c_{1}$ & $c_{2}$ & $c_{3}$ \\
\hline Dry asphalt & 1.2801 & 23.99 & 0.52 \\
Dry concrete & 1.1973 & 25.16 & 0.5373 \\
Wet asphalt & 0.857 & 33.82 & 0.347 \\
Snow & 1.946 & 94.12 & 0.0646 \\
Ice & 0.05 & 306.3 & 0 \\
\hline
\end{tabular}

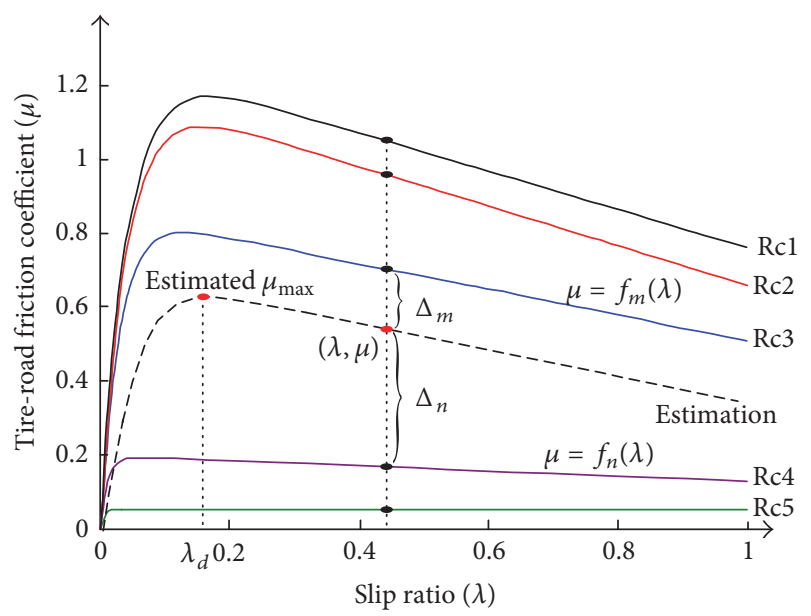

$\begin{array}{ll}\text { Rc: road condition } & \text { Rc 3: wet asphalt } \\ \text { Rc 1: dry asphalt } & \text { Rc 4: snow } \\ \text { Rc 2: dry concrete } & \text { Rc 5: ice }\end{array}$

FIGURE 2: Tire-road friction coefficient estimation [13].

In this paper, a widely used longitudinal $\mu$ - $\lambda$ relationship is adopted as

$$
\mu(\lambda)=c_{1}\left(1-e^{-c_{2} \lambda}\right)-c_{3} \lambda,
$$

where parameters of this tire model $c_{1}, c_{2}, c_{3}$ for different road surfaces are listed in Table 1 [15].

Table 1 shows five road conditions exactly, but in fact, the road condition is diverse, its instantaneous value may change, and therefore, this table can not be applied in all road condition. On this occasion, a simple and workable method based on these five road conditions and (7) is put forward.

The principle of tire-road friction coefficient estimation is shown as Figure 2. According to the instantaneous point $(\lambda, \mu)$ which is acquired from (2) to (5), compare the transient point $(\lambda, \mu)$ with each element of the five conditions and obtain a series of error values $\Delta_{i}$. Then, the two smallest error values $\Delta_{m}$ and $\Delta_{n}$ can be addressed; likewise the two curves $\mu=f_{m}(\lambda)$ and $\mu=f_{n}(\lambda)$ which are closest to $(\lambda, \mu)$ can be confirmed.

With the two error values, the real-time $\mu-\lambda$ relationship can be written as

$$
\mu(\lambda)=\frac{\Delta_{n}}{\Delta_{m}+\Delta_{n}} f_{m}(\lambda)+\frac{\Delta_{m}}{\Delta_{m}+\Delta_{n}} f_{n}(\lambda) .
$$

The maximum tire-road friction coefficient can be expressed as

$$
\mu_{\max }=f\left(\lambda_{d}\right) .
$$

It is worth noting that $\left(\lambda_{d}, \mu_{\max }\right)$ is the peak value point of (8); it can be addressed by taking the differential of (8). Where $\mu_{\max }$ is the maximum tire-road friction coefficient, $\lambda_{d}$ is the corresponding slip ratio of $\mu_{\max }$.

\section{MPC-Based Slip Controller Design}

In this section, a slip control strategy based on MPC is proposed. The primary task of MPC-based slip control system is to prevent the tires from locking up while braking, by decreasing the magnitude of the braking torque requested by the driver, to levels where the tire longitudinal force is maximized and the slip ratio is kept within a safe range that does not jeopardize the tire lateral forces. The slip control block diagram is shown in Figure 3 . Where $\lambda_{f l}, \lambda_{f r}, \lambda_{r l}, \lambda_{r r}$ are the four tires' slip ratio, $T_{b f l}, T_{b f r}, T_{b r l}, T_{b r r}$ are the braking torque of four tires.

As illustrated, it is shown above that according to the $\mu_{\max }$ estimation method and the five road conditions, the slip ratio equilibrium point must be addressed, which is the desired value for the control system. With the desired value of slip ratio and the observed value from vehicle model, the MPC-based slip controller can predict the future condition of the system and then output the optimized torque command to the vehicle model and control the real longitudinal slip ratio of wheels within the optimal one, while considering the actuators limitations, wheel slip constraints, and performance metrics. The real slip ratio of wheels and the vehicle velocity are feedback to the controller all the time.

4.1. Reference Slip Ratio. From the discussion above, it is obvious that the reference slip ratio varies for the difference of the tire peculiarities and the road adhesion conditions; therefore, to make the calculation and simulation more accurate, a real-time reference slip ratio must be given.

The reference slip ratio is shown in Figure 4. As illustrated, it can be found that the vehicle stays stable within a certain slip ratio value, and the highest friction coefficient exists when $\dot{\lambda}=0$, that is to say, $\lambda_{d}$ which has the maximum friction coefficient, that is, the maximum friction force [16]. The slip ratio equilibrium point is the desired value for the slip control system. Accordingly, during braking if slip ratio can be kept at $\lambda_{d}$ at all times ideally except for the timing of slip increasing from zero to $\lambda_{d}$ until the vehicle stops, the optimal brake performance can be achieved. For this purpose, $\lambda_{d}$ is defined as the reference slip ratio. The reference slip ratio, that is, the slip ratio equilibrium point, can be regarded as the desired value for the control system.

As with the ideal slip behavior mentioned above, the slip ratio can be maintained at $\lambda_{d}$ during braking. It is verifiable that if brake torque is controlled, the wheel is controlled in an ideal manner. Consequently, the wheel slip ratio does not become large and the wheel lock is avoidable.

4.2. Braking Torque Distribution between Front and Rear Wheel. When braking, the wheel dynamic load varies with deceleration, which leads the brake forces' change of both front and rear wheels. The higher the deceleration, the more the weight transference. Hence, appropriate brake force 


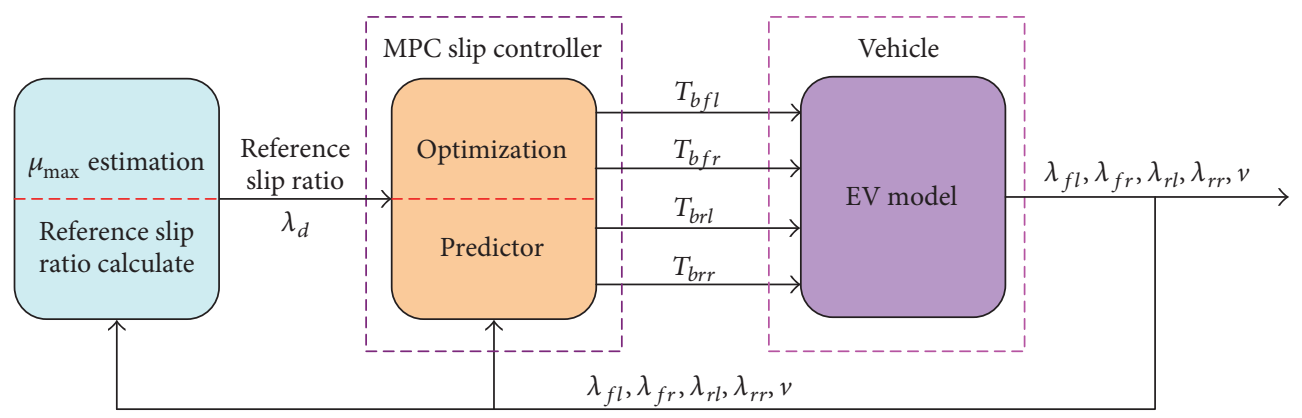

Figure 3: Block diagram of the MPC-based slip control system.

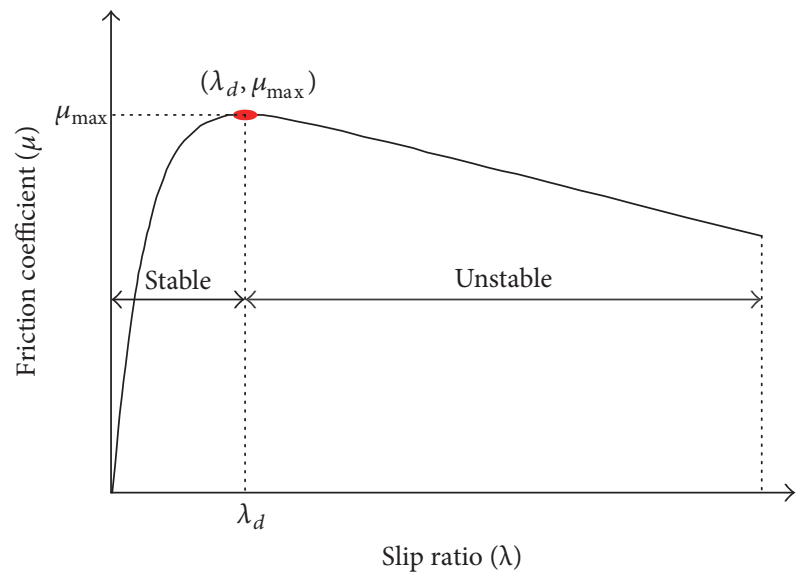

FIGURE 4: Reference slip ratio calculation.

design on the front and rear wheels is essential and required for the consideration of vehicle stability. In this paper, the distribution curve is denoted to be fixed value proportion. The distribution curve of fixed value proportion is the most widely used method of the real car because of its advantages of easy to implement and lower cost [17].

4.3. Slip Controller Design. Based on the slip control model (see (1)-(6)) of EV mentioned above, a state-space model for MPC can be obtained; then a finite dimensional optimal control problem can be defined [18]. Let the state-space model be derived as

$$
\begin{aligned}
x(k+1) & =A x(k)+B u(k), \\
y(k) & =C \cdot x(k),
\end{aligned}
$$

where $x=\left[\lambda_{f l}, \lambda_{f r}, \lambda_{r l}, \lambda_{r r}\right]^{T}$ is the state variable, $u=\left[T_{f l}\right.$, $\left.T_{f r}, T_{r l}, T_{r r}\right]^{T}$ is the control input, and $y=\left[\lambda_{f l}, \lambda_{f r}, \lambda_{r l}, \lambda_{r r}\right]^{T}$ is the system output.

Define $P$ as predictive horizon and $M$ as control horizon, $P \geq M \geq 1$. At the sampling instant, the control sequence $U_{k}$ which is the optimization vectors and system output sequence $Y_{k}$ is as follows [19]:

$$
\begin{gathered}
U_{k}=\left[\begin{array}{c}
u(k \mid k) \\
u(k+1 \mid k) \\
\vdots \\
u(k+M-1 \mid k)
\end{array}\right], \\
Y_{k}=\left[\begin{array}{c}
y(k+1 \mid k) \\
y(k+2 \mid k) \\
\vdots \\
y(k+P \mid k)
\end{array}\right] .
\end{gathered}
$$

At sample time $k, x(k)$ works as predictive origin which is equal to $x(k \mid k)$, and the predictive state and predictive output are calculated as follows [20,21]:

$$
\begin{array}{r}
x(k+i \mid k)=f(x(k+i-1 \mid k), u(k+i-1 \mid k)), \\
0 \leq i \leq M-1, \\
y(k+i \mid k) \\
=C \cdot(f(x(k+i-1 \mid k), u(k+i-1 \mid k))), \\
0 \leq i \leq P .
\end{array}
$$

Optimization variables are the torque command of the four wheels. So the optimization object function in this paper at step $k$ of the optimal control problem can then be written as

$$
\begin{aligned}
\min _{u} J & \\
= & \sum_{i=1}^{P}\left\|\Gamma_{y, i}(y(k+i \mid k)-r(k+i \mid k))\right\| \\
& +\sum_{i=1}^{M}\left\|\Gamma_{u, i} \Delta u(k+i-1 \mid k)\right\|,
\end{aligned}
$$

where $r(k+i)$ denotes the reference slip ratio of four wheels; $\Gamma_{y i}, \Gamma_{u i}$ are the weighting matrices. 


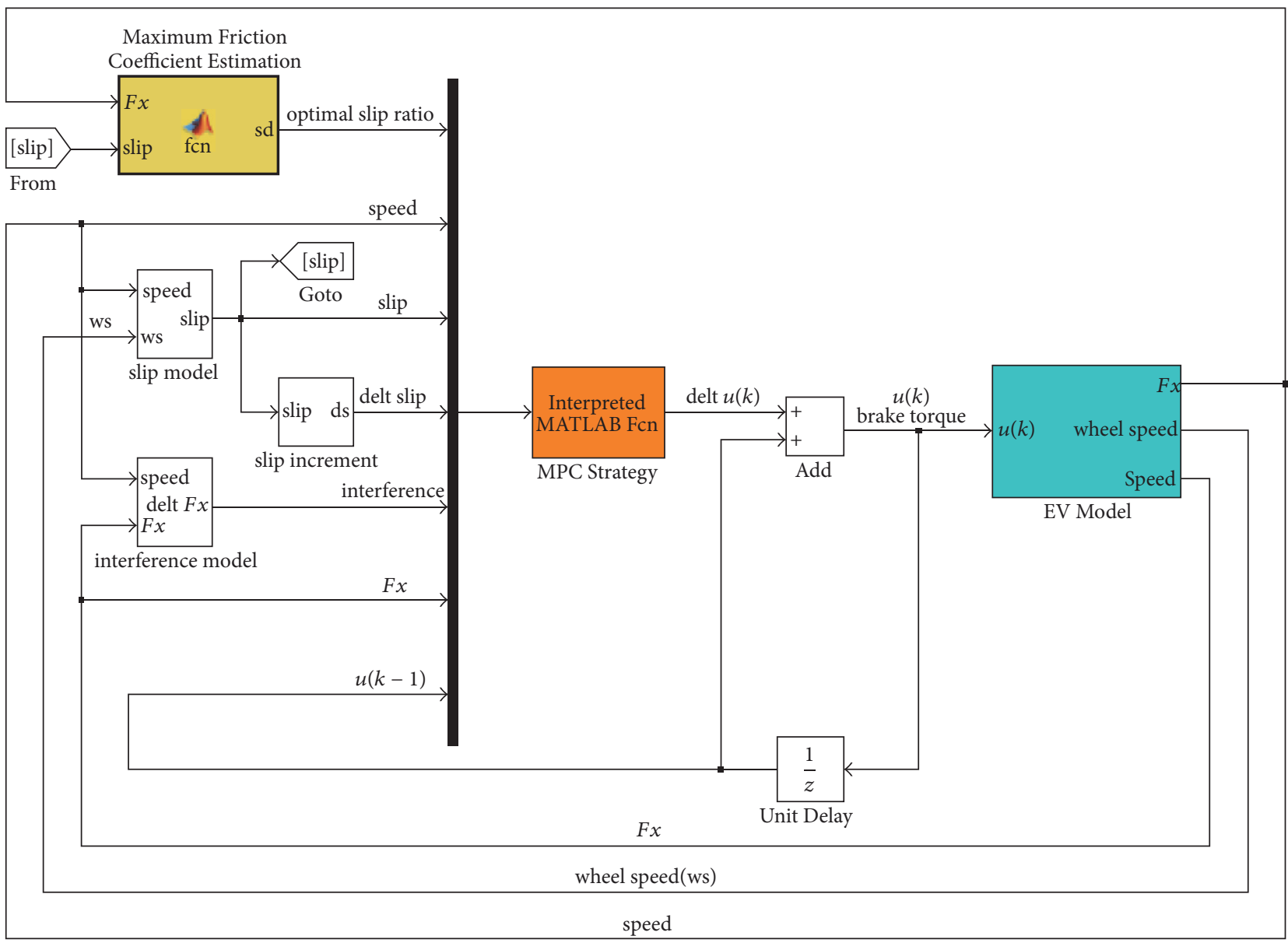

FIgURE 5: Slip control system based on estimation in Simulink.

$J_{1}=\sum_{i=1}^{P}\left\|\Gamma_{y, i}(y(k+i \mid k)-r(k+i \mid k))\right\|$ is to keep the real slip ratio of each wheel within the optimal one to prevent the tires from locking up.

$J_{2}=\sum_{i=1}^{M}\left\|\Gamma_{u, i} \Delta u(k+i-1 \mid k)\right\|$ is added to control the changing rate of the control action to preserve the comfort of $\mathrm{EV}$ driver, and the change rate of the control action should be small enough to ensure smooth braking of the vehicle.

MPC can effectively manage the multiple control requirements with the weighting matrix considered. Besides, MPC can also deal with the actuator constraint.

Take the motor maximum torque limitations and the value range of wheel slip into consideration, and the constrains can be written as follows:

$$
\begin{aligned}
\left|T_{i}(k+j \mid k)\right| & \leq T_{\max }, \quad j=0,1, \ldots, M-1 ; \\
0 & \leq \lambda_{i}(k+j \mid k) \leq 1, \quad j=0,1, \ldots, P .
\end{aligned}
$$

MPC solves online an optimization problem at each time instant, with knowledge of the current system state variables and previous system inputs, and then takes the first element of the optimal control sequence as the current control to the system.
TABLE 2: Main parameters of EV.

\begin{tabular}{lcc}
\hline Parameter & Symbol & Value \\
\hline Vehicle mass & $m$ & $1359.8 \mathrm{~kg}$ \\
Wheel inertia & $J$ & $0.82 \mathrm{~kg} \mathrm{~m}^{2}$ \\
Wheel radius & $R$ & $0.26 \mathrm{~m}$ \\
\hline
\end{tabular}

\section{Simulation and Analysis}

Simulations were conducted in the MATLAB/Simulink environment to show the control performance of the proposed controller. According to the engineering structure of $\mathrm{EV}$, a full-vehicle model was built in MATLAB/Simulink. The model mainly includes the powertrain model, the braking model, the steering model, the diver model, the tire model, the road-wheel model, and so on. The main parameters of EV are shown in Table 2. The proposed controller of one wheel in Simulink is shown in Figure 5. In this model, the slip controller can respectively control the four wheels at the same time. Besides, the real longitudinal slip ratio of the four wheels is calculated and feedback to the controller from the EV model.

In this simulation, the simulation step is defined as $0.001 \mathrm{~s}$, the predictive horizon $P=10$, and the control horizon 


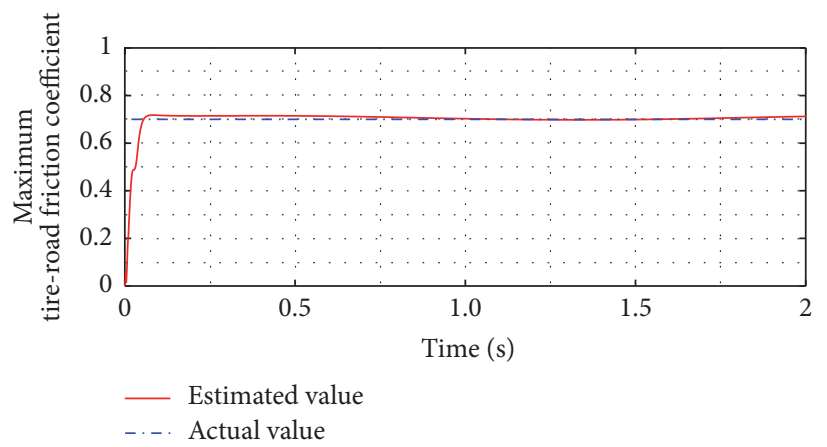

(a) Maximum tire-road friction coefficient

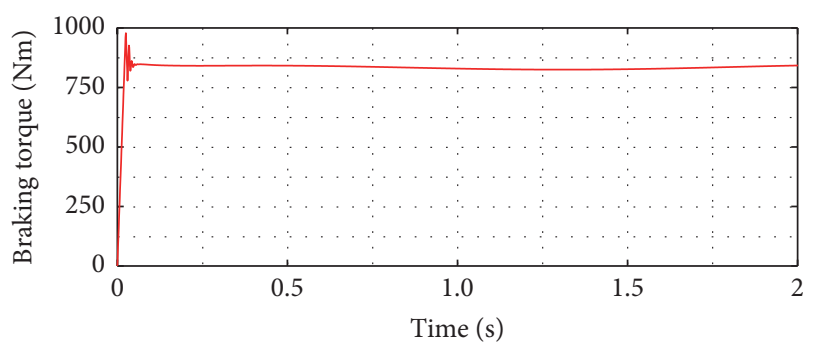

(c) Braking torque

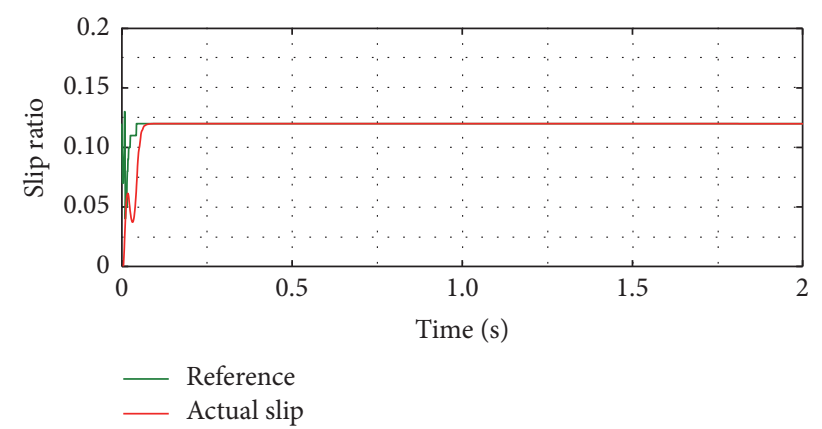

(b) Slip ratio

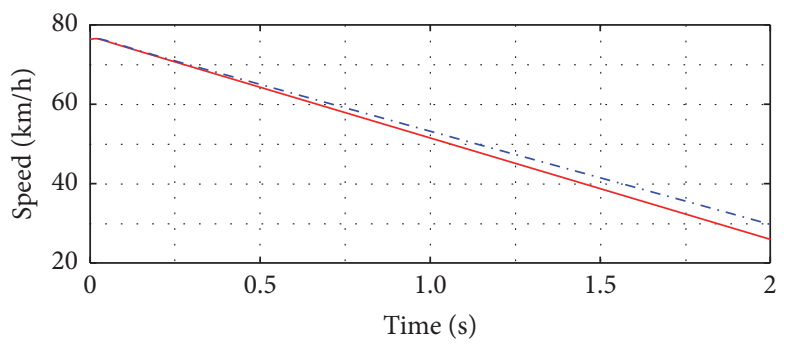

- MPC-control

-... Noncontrol

(d) Vehicle speed

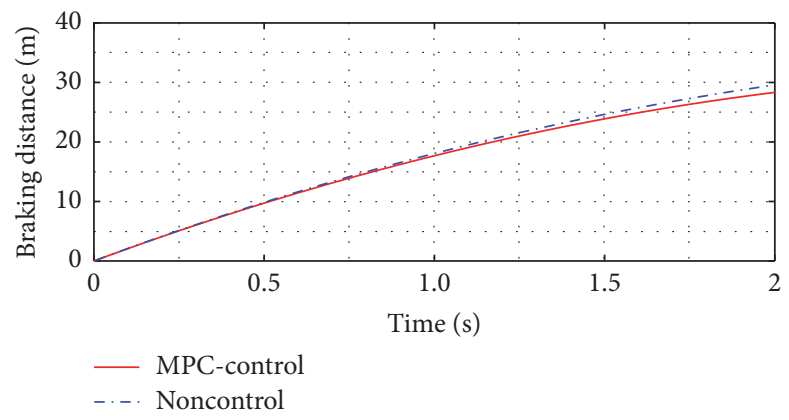

(e) Braking distance

FIGURE 6: Simulation results of vehicle braking while road adhesion coefficient is 0.7 . Red curve refers to braking torque.

$M=5$. Two conditions which have different road adhesion coefficients are set to verify the performance of proposed controller. The initial speeds under the two conditions are both determined to $78 \mathrm{~km} / \mathrm{h}$, and the road adhesion coefficients are set to 0.7 and 0.25 , respectively. Figure 6 shows the state responses of simulation while the road adhesion coefficient is 0.7. As can be seen from this figure, the maximum tireroad friction coefficient $\left(\mu_{\max }\right)$ estimated by the controller designed in this paper can generally track the actual value. Furthermore, from the response of slip ratio, it can be seen that, at the first $0.1 \mathrm{~s}$, the reference slip ratio has a little fluctuation because of the sudden brake; the sudden brake leads to the mutation of the actual slip ratio and makes the reference slip ratio wave. Then, the reference slip ratio tends to stability and remains at 0.12 . The slip ratio controlled by the controller tracks the reference slip ratio well in this critical condition. From graph (c) of Figure 6, the braking torque shows a sharp increase to the peak value $1000 \mathrm{Nm}$; then it declines and achieves stability. Because of the sharp increase of slip ratio, the braking torque reaches the maximum value quickly, and then when the slip ratio tends to be stable, the braking torque calculated by MPC becomes stable finally. In addition, graph (d) and graph (e) show a high performance of the proposed MPC controller by contrast with simulation result of nonproposed controller. As illustrated, the speed of proposed MPC controller decreases faster than simulation of nonproposed controller. Figure 7 shows the responses while the road adhesion coefficient is 0.25 . As can be seen, Figure 7 has a similar tendency with Figure 6, except for the difference of the values. In addition, the two braking distances under the proposed controller in Figures 6 and 7 are much shorter compared with the braking distances 


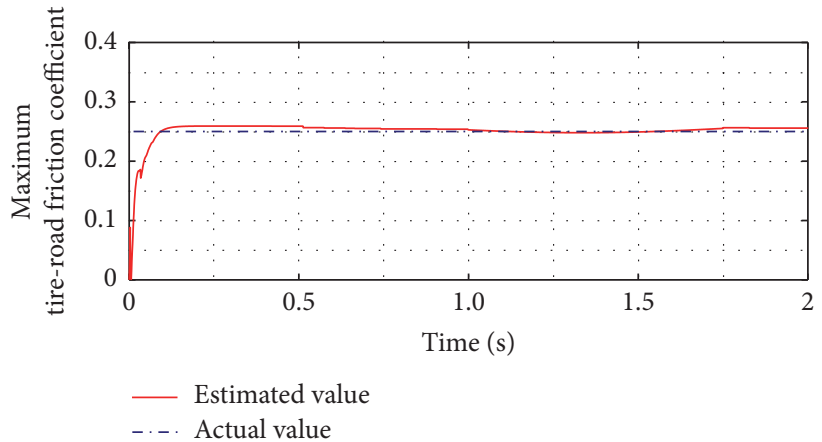

(a) Maximum tire-road friction coefficient

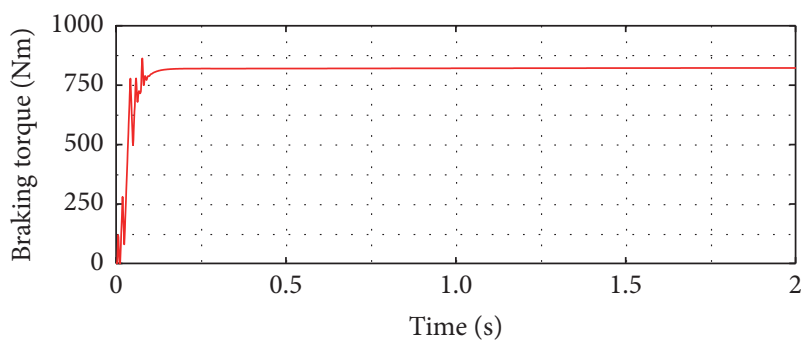

(c) Braking torque

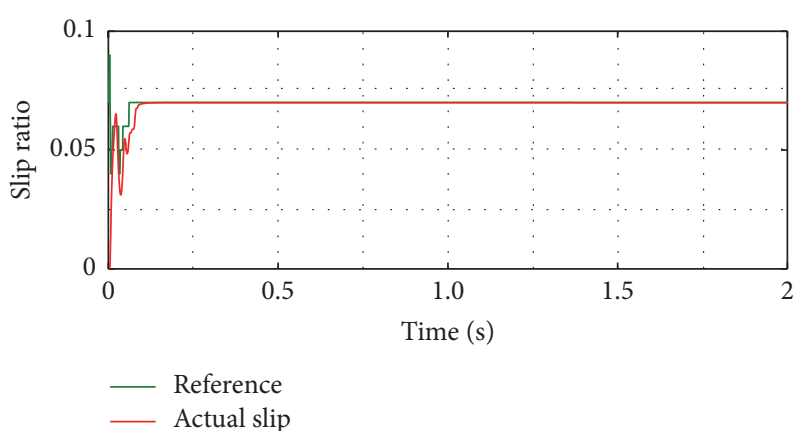

(b) Slip ratio

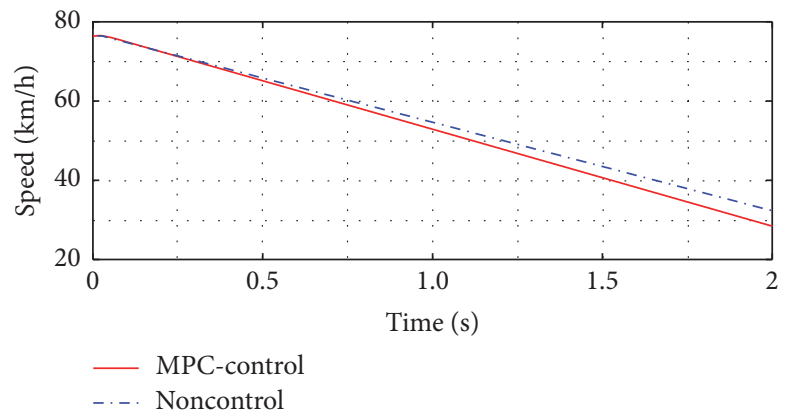

(d) Vehicle speed

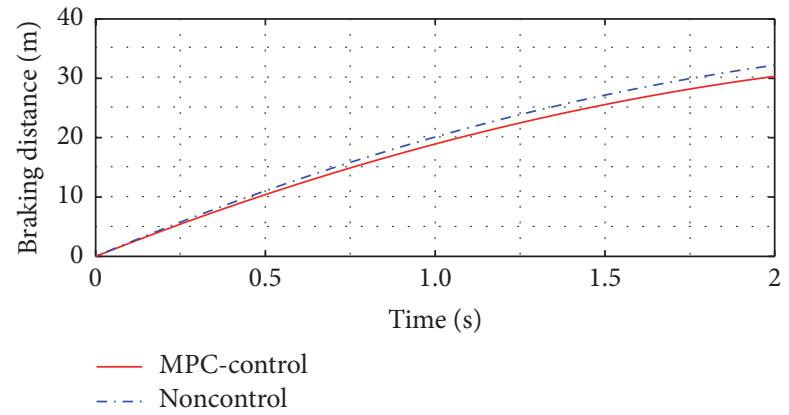

(e) Braking distance

FIGURE 7: Simulation results of vehicle braking while road adhesion coefficient is 0.25 . Red curve refers to braking torque.

and, under the non-MPC control system, this proofs that the braking performance under the proposed controller is much better than the non-MPC control system.

To sum up, the proposed controller estimates the realtime maximum tire-road friction coefficient under various road adhesion coefficient conditions, defines an instantaneous reference slip ratio, and guarantees the optimal braking torque on each wheel by individually controlling the slip ratio of each tire within the stable zone with a much faster response time; it can prevent the wheel from locking up and have a good performance while braking on various road conditions.

\section{Conclusion}

The slip control system based on tire-road friction coefficient estimation was presented in this paper to prevent vehicles from locking up in various road conditions. This proposed control system estimates the maximum tire-road friction in real-time under various road conditions and accurately controls the longitudinal slip ratio of the four wheels within the optimal one. Moreover, the control strategy makes full use of motor braking considering maximum capacity of motor so that the superior energy efficiency is expected as well. The effectiveness of the proposed control systems has been verified through simulations using an EV model which was built in Simulink. Several simulation studies were carried out. The results simultaneously compared with an uncontrolled system demonstrate that the braking performance of the EV was significantly improved by the proposed control system.

\section{Conflicts of Interest}

The authors declare that they have no conflicts of interest.

\section{Acknowledgments}

The work is supported by the Jilin Province Science and Technology Fund for Young Scholars (20170520097JH; no. 
20160520106JH), by Key Technology on Major Program of Jilin Province (20170201005GX), and by the Ministry of Education "Chunhui Plan” (Z2016020).

\section{References}

[1] A. Kihm and S. Trommer, "The new car market for electric vehicles and the potential for fuel substitution," Energy Policy, vol. 73, pp. 147-157, 2014.

[2] B. Wang, X. Huang, J. Wang, X. Guo, and X. Zhu, "A robust wheel slip ratio control design combining hydraulic and regenerative braking systems for in-wheel-motors-driven electric Vehicles," Journal of The Franklin Institute, vol. 352, no. 2, pp. 577-602, 2015.

[3] B. K. Dash and B. Subudhi, "Effects of sliding surface on the performances of adaptive sliding mode slip ratio controller for a HEV," Archives of Control Sciences, vol. 23, no. 2, pp. 187-203, 2013.

[4] H. Gao, K. Xia, L. Ding, Z. Deng, Z. Liu, and G. Liu, "Optimized control for longitudinal slip ratio with reduced energy consumption," Acta Astronautica, vol. 115, pp. 1-17, 2015.

[5] J. Wang, L. Alexander, and R. Rajamani, "Friction estimation on highway vehicles using longitudinal measurements," Journal of Dynamic Systems, Measurement, and Control, vol. 126, no. 2, pp. 265-275, 2004.

[6] U. Takaji, "Estimation of Tire-road Friction by Tire Rotational Vibration Model," RD Review of Toyota CRDL, vol. 37, 3, pp. 53$58,2002$.

[7] M. A. Wilkin, W. J. Manning, D. A. Crolla, and M. C. Levesley, "Use of an extended Kalman filter as a robust tyre force estimator," Vehicle System Dynamics, vol. 44, no. 1, pp. 50-59, 2006.

[8] V. Ivanovic, J. Deur, M. Kostelac et al., "Experimental identification of dynamic tire friction potential on ice surfaces," Vehicle System Dynamics, vol. 44, no. 1, pp. 93-103, 2006.

[9] X. Yang, "Improvements in vehicle handling and stability by a novel wheel slip coordination control scheme," International Journal of Vehicle Design, vol. 62, no. 2-4, pp. 206-230, 2013.

[10] R. De Castro, R. E. Araújo, M. Tanelli, S. M. Savaresi, and D. Freitas, "Torque blending and wheel slip control in EVs with in-wheel motors," Vehicle System Dynamics, vol. 50, no. 1, pp. 71-94, 2012.

[11] W. Chiang, D. Yin, M. Omae, and H. Shimizu, "Integrated SlipBased Torque Control of Antilock Braking System for In-Wheel Motor Electric Vehicle," IEEJ Journal of Industry Applications, vol. 3, no. 4, pp. 318-327, 2014.

[12] G. Liu and L. Jin, "A Study of Coordinated Vehicle Traction Control System Based on Optimal Slip Ratio Algorithm," Mathematical Problems in Engineering, vol. 2016, Article ID 3413624, 2016.

[13] M. Bian, L. Chen, Y. Luo, and K. Li, "Research on maximum road adhesion coefficient estimation for distributed drive electric vehicle," in Proceedings of the 2013 International Conference on Mechanical and Automation Engineering, MAEE 2013, pp. 90-94, Jiujang, China, July 2013.

[14] J. Peng, H. He, and N. Feng, "Simulation research on an electric vehicle chassis system based on a collaborative control system," Energies, vol. 6, no. 1, pp. 312-328, 2013.

[15] R. Bhandari, S. Patil, and R. K. Singh, "Surface prediction and control algorithms for anti-lock brake system," Transportation Research Part C: Emerging Technologies, vol. 21, no. 1, pp. 181195, 2012.
[16] E. Hashemi, A. Kasaiezadeh, S. Khosravani, A. Khajepour, N. Moshchuk, and S.-K. Chen, "Estimation of longitudinal speed robust to road conditions for ground vehicles," Vehicle System Dynamics, vol. 54, no. 8, pp. 1120-1146, 2016.

[17] K. Maeda, H. Fujimoto, and Y. Hori, "Four-wheel driving-force distribution method for instantaneous or split slippery roads for electric vehicle," Automatika - Journal for Control, Measurement, Electronics, Computing and Communications, vol. 54, no. 1, pp. 103-113, 2013.

[18] L. Yuan, H. Chen, B. Ren, and H. Zhao, "Model predictive slip control for electric vehicle with four in-wheel motors," in Proceedings of the 34th Chinese Control Conference, CCC 2015, pp. 7895-7900, Hangzhou, China, July 2015.

[19] H. Zhao, B. Gao, B. Ren, H. Chen, and W. Deng, "Model predictive control allocation for stability improvement of fourwheel drive electric vehicles in critical driving condition," IET Control Theory \& Applications, vol. 9, no. 18, pp. 2688-2696, 2015.

[20] B. Ren, H. Chen, H. Zhao, and L. Yuan, "MPC-based yaw stability control in in-wheel-motored EV via active front steering and motor torque distribution," Mechatronics, vol. 38, pp. 103114, 2016.

[21] L. Yuan, H. Zhao, H. Chen, and B. Ren, "Nonlinear MPC-based slip control for electric vehicles with vehicle safety constraints," Mechatronics, vol. 38, pp. 1-15, 2016. 


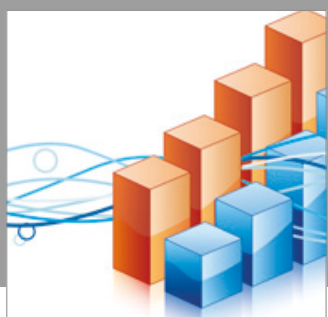

Advances in

Operations Research

vatersals

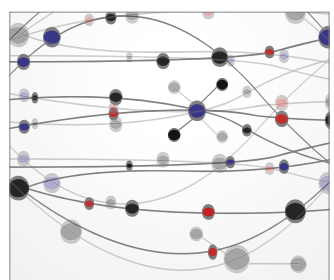

\section{The Scientific} World Journal
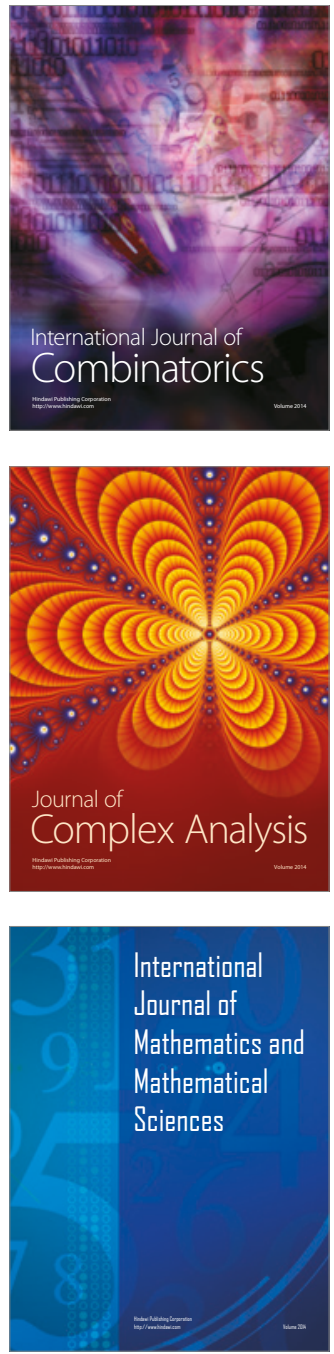
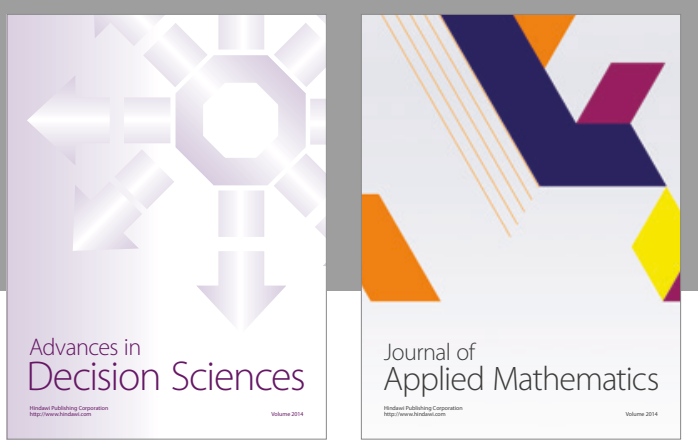

Algebra

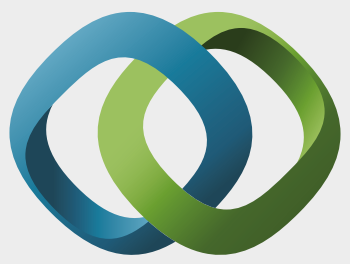

\section{Hindawi}

Submit your manuscripts at

https://www.hindawi.com
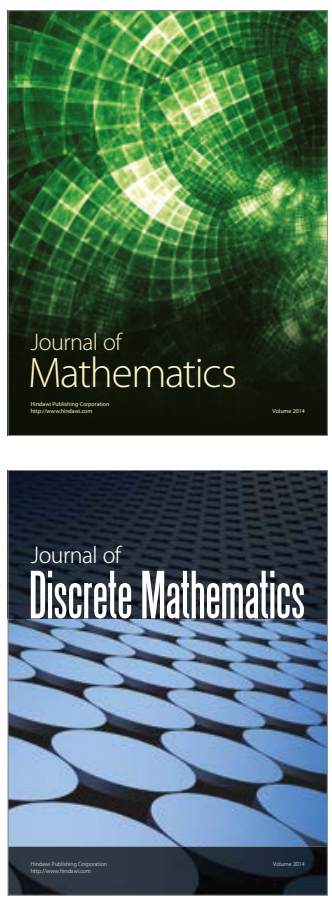

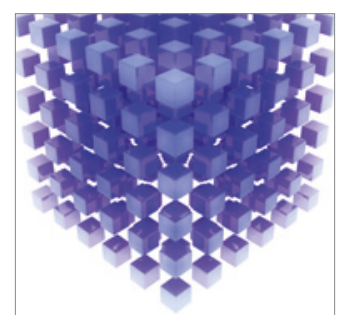

Mathematical Problems in Engineering
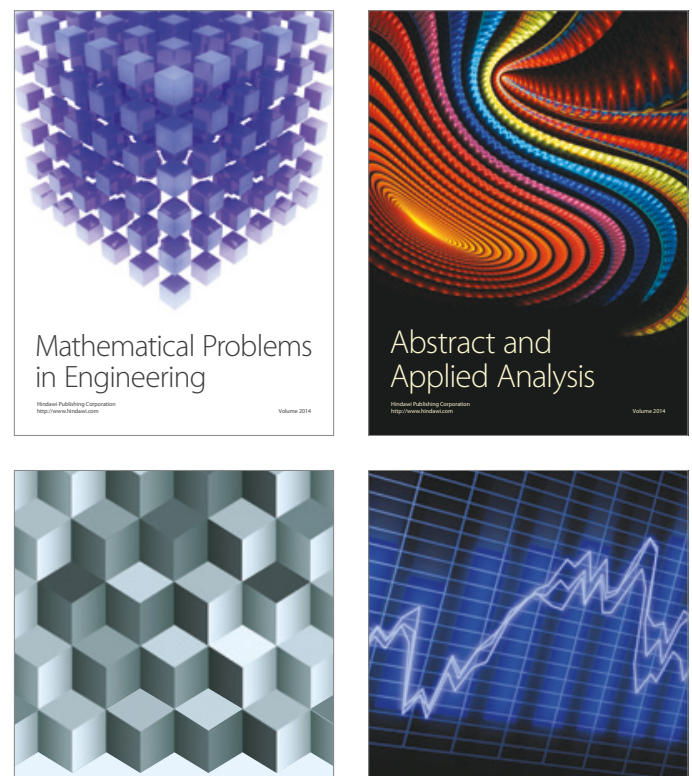

Journal of

Function Spaces

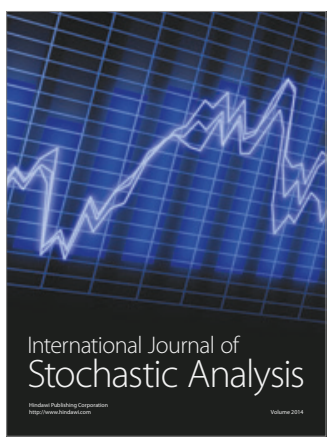

Probability and Statistics
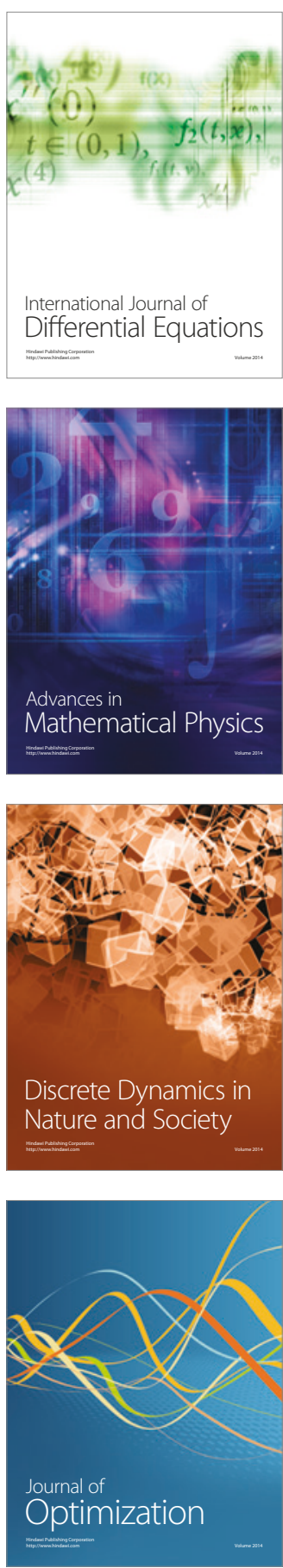